\title{
Delayed Facial Nerve Paralysis after Vestibular Schwannoma Resection
}

\author{
Robert J. Yawn ${ }^{1}$ Matthew M. Dedmon ${ }^{1}$ Deborah Xie ${ }^{1}$ Reid C. Thompson ${ }^{2}$ Matthew R. O'Malley ${ }^{1}$ \\ Marc L. Bennett ${ }^{1} \quad$ Alejandro Rivas ${ }^{1}$ David S. Haynes ${ }^{1}$
}

1 Department of Otolaryngology, Vanderbilt University Medical Center, Nashville, Tennessee, United States

2 Department of Neurologic Surgery, Vanderbilt University Medical Center, Nashville, Tennessee, United States

J Neurol Surg B 2019;80:283-286.
Address for correspondence David S. Haynes, MD, MMHC, Department of Otolaryngology-Head and Neck Surgery, Vanderbilt University Medical Center, 7209 Medical Center East-South Tower, 1215 21st Avenue. South, Nashville, TN 37232-8605, United States (e-mail: david.haynes@vanderbilt.edu).

\begin{abstract}
Keywords

- vestibular schwannoma

- acoustic neuroma

- facial paralysis

- skull base surgery

- translabyrinthine

- suboccipital

- retrosigmoid

- delayed facial paralysis

Objective To describe the incidence and clinical course of patients who develop delayed facial nerve paralysis (DFNP) after surgical resection of vestibular schwannoma.

Setting Tertiary skull base center.

Methods Retrospective chart review.

Results Two hundred and forty six consecutive patients, who underwent surgical resection for vestibular schwannoma at a single center between 2010 and 2015, were analyzed. Of these patients, 22 (8.9\%) developed DFNP, defined here as deterioration of function by at least 2 House-Brackmann (HB) grades within 30 days in patients with immediate postoperative $\mathrm{HB} \leq 3$. The mean age of DFNP patients was 47.2 years (range: $17-67)$ and 16 (73\%) were female. The mean tumor size in greatest dimension was $2.1 \mathrm{~cm}$ (range: $0.7-3.5 \mathrm{~cm}$ ). At the conclusion of each case, the facial nerve stimulated at the brainstem. Mean immediate postoperative facial nerve function was HB 1.8 (range: $1-3$ ). Average facial nerve function at the 3-week-postoperative visit was 4.4 (range: $2-6$ ). In 1-year, 8 patients (36\%) recovered HB 1 function, 10 patients (46\%) recovered to $\mathrm{HB} 2$, and 2 patients $(9 \%)$ were $\mathrm{HB} 3$. The remaining 2 patients did not recover function and were $\mathrm{HB} 6$ at last follow-up. Initial postoperative facial nerve function (HB 1 or HB 2) was associated with improved recovery to normal (HB 1) function $(p=0.018)$.

Conclusion A majority of patients that develop delayed paralysis will recover excellent facial nerve function. Patients should be counseled; however, a small percentage of patients will not recover function long-term, despite having a previously functioning and anatomically intact nerve.
\end{abstract}

\section{Introduction}

Facial nerve paresis or paralysis is one of the most clinically important morbidities associated with vestibular schwannoma surgery. As surgical techniques have advanced, facial nerve outcomes continue to improve. Outcomes in patients that have anatomically disrupted nerves and undergo cable grafting or split-nerve grafting are understandably poor and a have a ceiling with regards to nerve function, with most outcomes clustering around House-Brackmann (HB) 3 or 4. ${ }^{1,2} \mathrm{~A}$ subset of patients exists with a normal functioning, or near-normal functioning nerve in the immediate postoperative period that has subsequent deterioration of function (i.e., delayed facial nerve paralysis [DFNP]). received

March 5, 2018

accepted

July 17, 2018

published online

September 6, 2018 (c) 2019 Georg Thieme Verlag KG Stuttgart - New York
DOI https://doi.org/ 10.1055/s-0038-1669941. ISSN 2193-6331. 
Delayed facial nerve paralysis has been described in a variety of clinical settings, including temporal bone fracture, blast injury, and after surgical interventions that take place in close proximity to the nerve throughout its course. ${ }^{3,4}$ These surgeries can include vestibular schwannoma surgery, dental procedures, maxillofacial trauma repair, and tympanomastoid surgery. ${ }^{5-8}$ While the exact mechanism of DFNP is unknown, theories regarding the pathogenesis include postoperative inflammation, vascular spasm, and viral reactivation. ${ }^{9,10}$ As such, many clinicians prefer to treat this entity with a combination of antiviral medications and glucocorticoids, although studies have shown that patients, who undergo no additional treatment have similar outcomes to those that undergo either steroid therapy or steroid and antiviral therapy. ${ }^{11}$ Further complicating the treatment algorithm is the observation that many patients with DFNP after vestibular schwannoma resection have onset of symptoms following discharge from the hospital, making diagnosis and treatment difficult in the postoperative setting.

Previous reports of DFNP in the setting of vestibular schwannoma surgery describe an incidence between 16 and 25\% of cases with excellent prognosis. ${ }^{11,12}$ In the series reported by Carlstrom and colleagues all patients recovered to HB 1 or HB 2 by a mean of 33 days. ${ }^{11}$ Through the authors' experience; however, a small subset of patients with DFNP was identified with poor return of function. The purpose of this study was to further describe and characterize DFNP after vestibular schwannoma resection, with additional emphasis placed on patients that do not recover excellent facial nerve function.

\section{Methods}

After institutional review board approval, a retrospective review was performed to identify all patients that underwent vestibular schwannoma resection at a single tertiary skull base center between 2010 and 2015. A radiologic diagnosis was made based on the specific magnetic resonance imaging (MRI) characteristics of vestibular schwannoma and the diagnosis was confirmed on histopathologic analysis. Only patients with histopathologically confirmed World Health Organization (WHO) grade I schwannoma were included for analysis. Patient demographics and tumor characteristics were recorded from electronic medical records. Tumor dimensions were defined as the greatest linear dimension in the axial plane. Facial nerve outcomes were described and characterized using the $\mathrm{HB}$ scale. $^{13}$ DFNP was defined in this study as patients with immediate postoperative documented $\mathrm{HB} \leq 3$ with subsequent deterioration of at least $2 \mathrm{HB}$ grades within 30 days, postoperatively. All patients underwent either translabyrinthine or retrosigmoid excision and underwent a postoperative steroid taper as a part of our center's craniotomy pathway. Gross total resection was defined as entire tumor removal. Near total resection was defined as known tumor that was intentionally left on a critical neurovascular structure but not visible on postoperative MRI, and subtotal resection was defined as known tumor left behind to preserve critical neurovascular structures that was visible on postoperative MRI. Only patients with an anatomically intact and electrically stimulating nerve were included. Patients were excluded if they had insufficient follow-up data. The primary outcome measure was $\mathrm{HB}$ grade at 1-year postoperative follow-up. A secondary outcome measure was the development of synkinesis.

A secondary analysis was performed dividing DFNP patients into 2 groups: patients that had early deterioration of nerve function with onset of delayed paralysis in the first 5 days after surgery, and those that had late deterioration with loss of function after the first 5 days after surgery.

Features were summarized with means, medians, ranges, and standard deviations (SD), where applicable. Log-Rank (Mantel-Cox) test was used to compare evaluate the return of function over time. Mann-Whitney tests were used to compare continuous variables. 2-way ANOVA (analysis of variance) was used to compare early and late DFNP facial nerve function. Fisher's exact tests were used to compare categorical data. Statistical analyses were performed using Graphpad Prism (La Jolla, CA, the U.S.).

\section{Results}

\section{Patient Demographics and Clinical Details}

Two hundred and forty six consecutive patients were initially analyzed, who underwent surgical excision for histopathologically confirmed vestibular schwannoma between 2010 and 2015. Of these patients, 22 (8.9\%) developed DFNP, defined here as deterioration of function by at least $2 \mathrm{HB}$ grades within 30 days in patients with immediate postoperative $\mathrm{HB} \leq 3$. The mean age of DFNP patients was 47.2 years (range: $17-67$ ) and 16 (73\%) were female. The primary presenting symptom in all but 1 patient was asymmetric hearing loss with the other patients' primary presenting symptom, being vertigo. Two patients (9\%) had comorbid diabetes mellitus and 8 patients (36\%) had comorbid hypertension. Two patients ( $9 \%$ ) were actively using tobacco at the time of surgery, and 2 patients (9\%) were previous tobacco users. There were no significant differences in rates of hypertension, diabetes mellitus, or tobacco abuse in patients that developed DFNP versus those that did not.

The mean tumor size in the greatest dimension was $2.1 \mathrm{~cm}$ (range: $0.7-3.5 \mathrm{~cm}$ ). The majority of DFNP patients underwent translabyrinthine excision of their tumors ( $n=19,86 \%$ ), with the remaining patients undergoing retrosigmoid approach for tumor excision $(n=3,14 \%)$. All patients had an anatomically intact nerve at the conclusion of the case that electrically stimulated at $0.05 \mathrm{~mA}$ at the brainstem. Sixteen patients (73\%) underwent gross total resection, 5 patients (23\%) underwent near total resection, and 1 patient (4\%) underwent subtotal resection. Two patients that underwent near total resection and the 1 patient that underwent subtotal resection, had tumor recurrence, or progression of residual tumor requiring stereotactic radiosurgery.

\section{Facial Nerve Outcomes}

All patients had normal (HB 1) facial nerve function preoperatively with the exception of 1 patient, who had mild (HB 2) facial nerve paresis. Immediate postoperative facial 




Fig. 1 Graphical depiction of the clinical course of patients with delayed facial nerve paralysis.

nerve function (documented either the evening of postoperative day 0 or the morning of postoperative day 1) was HB 1 in 10 patients (45\%), HB 2 in 5 patients (23\%) and $\mathrm{HB} 3$ in 7 patients (32\%). The mean immediate postoperative facial nerve function was HB 1.8 (range: 1-3). Average facial nerve function at the 3-week-postoperative visit was 4.4 (range: $2-6$ ). In 1 -year, 8 patients (36\%) recovered HB 1 function, 10 patients (46\%) recovered to HB 2 , and 2 patients $(9 \%)$ were HB 3 . The remaining 2 patients did not recover function and were HB 6 at last follow-up. Both patients that were HB 6 at last follow-up, had immediate postoperative facial nerve function of $\mathrm{HB} 3$. The clinical course of DFNP patients is graphically depicted in - Fig. 1. Initial postoperative facial nerve function (HB 1 or $\mathrm{HB} 2$ ) was associated with improved recovery to normal (HB 1) function $(p=0.018)$. No patient with initial postoperative HB 3 that had DFNP recovered to HB 1. In 1-year follow-up, 8 patients (36\%) were HB 1, 10 patients (45\%) were HB 2, 2 patients (9\%) were HB 3, and 2 patients (9\%) were HB 6. Six patients (27\%) developed synkinesis.

\section{Early Versus Late Onset DFNP}

Of the 22 patients that developed DFNP, 14 patients (64\%) had early deterioration of function (within the first 5 days after surgery) and 8 patients (36\%) had late deterioration (between 5-30 days postoperatively). The mean age of early DFNP patients was 44.4 years (range: $17-60$ years) versus 52.3 years (range: $27-67$ years) in the late DFNP group $(p=0.21)$. There were no significant differences in rates of diabetes mellitus, hypertension, or tobacco use in the 2 groups. The mean tumor size was $2.4 \mathrm{~cm}$ (range: $0.7-3.5 \mathrm{~cm}$ ) in the early DFNP versus $1.5 \mathrm{~cm}$ (range: $0.7-1.9 \mathrm{~cm}$ ) in the late DFNP group $(p=0.012$ ). In 1-year follow-up, all of the patients in the late DFNP group recovered to HB 1 or HB 2 function. The mean facial nerve function of patients with early deterioration was 2.43 at 1 -year (median: 2, range: $1-6$ ) versus 1.5 -year (median: 1.5 ; range: $1-2 ; p=0.11)$.

\section{Discussion}

Herein, the authors describe a series of patients, where delayed facial nerve paralysis was observed after vestibular schwannoma excision via traditional skull base surgical approaches. Prior studies have shown the incidence of DFNP to be estimated to be between 16 and $25 \%{ }^{11,12}$ The incidence in this series was lower than these reports at $8.9 \%$.

While this is not the first description of delayed facial nerve paralysis in the literature, the study does show that within this specific series, the clinical outcomes have the potential for more variability than previously described. Specifically, the authors propose that recovery in some patients to normal or near-normal function may take longer than previously reported, and that a small proportion of patients may not experience recovery.

In a recent report by Carlstrom and colleagues, discussing this phenomenon in 60 patients, all patients recovered to HB 1 or HB 2 by a mean of 33 days (range: 7-86 days). By comparison, in our series, most patients achieved normal or near normal (HB 1 or HB 2) function, but the recovery time was often longer with recovery of function, typically not documented until the 12-month follow-up visit. Considerable variability in function was observed at the 3-month postoperative visit, as depicted in - Fig. 1. Furthermore, no patients in the previously mentioned study had poor ( $\mathrm{HB} 4$ or greater) function long-term. In this study, 2 patients had postoperative deterioration of $\mathrm{HB} 3$ function to $\mathrm{HB} 6$ function and never regained facial nerve function, eventually undergoing static reanimation for restoration of function. These patients both underwent a translabyrinthine excision for tumors that were 2.0 and $3.2 \mathrm{~cm}$. There were no postoperative complications in either patient and both nerves electrically stimulated at the end of the case. Both patients had facial nerve paresis (HB 3) in the immediate postoperative period. While the above study was not designed to investigate the etiology of their poor outcome, the authors suspect that the postoperative phenomena (inflammation or vascular) that are hypothesized to result in DFNP, may have a worse impact in a nerve that is already "threatened" and demonstrating decreased postoperative function.

When the patients were divided into subgroups based on the onset of DFNP, there was a trend toward worse function in patients that had early onset of DFNP (within 5 days, postoperatively) versus those that had late onset (between 5-30 days, postoperatively). In this small series; however, this trend was not statistically significant. It is worth noting that all of the patients that had late DFNP recovered to HB 1 or HB 2 in 1 -year. These results are more consistent with the favorable outcomes seen in the recent report by Carlstrom and colleagues. ${ }^{11}$ While the study is not designed to understand the pathogenesis of these patients, the authors hypothesize that the late onset DFNP may be more related to viral reactivation, rather than postoperative inflammation seen in the early onset group. However, both groups were only treated with oral glucocorticoids as a part of a postoperative craniotomy pathway. It is also worth noting that patients that had early DFNP did have larger tumors, which may also be influencing this series. 
Previous studies have described meatal or labyrinthine decompression of the facial nerve at the time of surgery to prevent DFNP after vestibular schwannoma surgery. ${ }^{14,15}$ While these studies suggest a decreased incidence of this phenomenon in patients that have pre-emptive decompression, it is not the routine protocol at the authors' center. Other centers have investigated the use of nimodipine, a calcium channel blocking medication, as a prophylactic neural protective agent in vestibular schwannoma surgery. A pilot study by Scheller and colleagues suggested that it might have a protective effect, when given prophylactically. ${ }^{16}$ Furthermore, another study by the same group noted that DFNP developed in patients, undergoing nimodipine treatment after vestibular schwannoma excision after the conclusion of treatment with the medication. ${ }^{10}$ In these patients, nimodipine was restarted and the DFNP resolved, suggesting a vascular etiology in these cases.

This study has several limitations. As a retrospective review of the medical record, there are inherent limitations and conclusions should be interpreted with caution. Additionally, while the HB facial nerve grading system is used in the literature as an objective assessment of facial nerve function, there is known interobserver variability, when used as a clinical assessment. This variability is typically minimal with normal or near normal function, but is seen in greater degree with increasing levels of facial nerve impairment. ${ }^{17}$ Furthermore, there is no consensus definition of DFNP, leading to heterogeneity within the literature. Most studies define it as deterioration within 30 days postoperatively, but there is considerable variation and substratification that can make it difficult to compare the outcomes from study to study. For example, the study by Magliulo and colleagues in 2003, included all patients that developed delayed paralysis and categorized them into categories based on the onset of symptoms after surgery (1-5 days, $6-14$ days, > 14 days). ${ }^{8}$ A study, by Franco-Vidal and colleagues, defined it as deterioration of function 72 hours after surgery. ${ }^{9}$ A later study, by Morton and colleagues, defined DFNP as deterioration of function within the first 30 days after surgery with normal postoperative function. This study also subdivided groups into late-onset DFNP ( $>72$ hours) and 'deteriorators,' defined as those that underwent further deterioration of function after initial onset of DFNP. ${ }^{12}$ Carlstrom and colleagues defined delayed facial palsy as deterioration in facial function of at least $2 \mathrm{HB}$ grades between postoperative days from 5 to $30 .^{11}$ These examples highlight the need for a consensus on the definition for future clinical studies.

\section{Conclusion}

DFNP is a commonly described phenomenon, following vestibular schwannoma excision. Recovery of normal or near-normal function is expected and a generally favorable prognosis should be communicated with patients. There is a small subset of patients, typically those with reduced immediate postoperative function that has worse longterm function. This may impact postoperative counseling in patients with this phenomenon.
Institutional Review Board Approval

Vanderbilt University IRB 32 approval 171083.

Financial Material and Support

No funding to disclose.

\section{Conflict(s) of Interest to Declare}

David S. Haynes is a consultant for Advanced Bionics Corporation, Cochlear Corporation, and MED-EL GmbH.

\section{References}

1 Prasad SC, Balasubramanian K, Piccirillo E, et al. Surgical technique and results of cable graft interpositioning of the facial nerve in lateral skull base surgeries: experience with 213 consecutive cases. J Neurosurg 2018;128(02):631-638

2 Yawn RJ, Wright HV, Francis DO, Stephan S, Bennett ML. Facial nerve repair after operative injury: Impact of timing on hypoglossal-facial nerve graft outcomes. Am J Otolaryngol 2016;37(06):493-496

3 Li Q Jia Y, Feng Q et al. Clinical features and outcomes of delayed facial palsy after head trauma. Auris Nasus Larynx 2016;43(05):514-517

4 Tripathi PB, Floriolli D, Caughlin BP. Delayed facial nerve paralysis following blast trauma. Facial Plast Surg 2017;33(01):116-118

5 Gaudin RA, Remenschneider AK, Phillips K, et al. Facial palsy after dental procedures - Is viral reactivation responsible? J Craniomaxillofac Surg 2017;45(01):71-75

6 Manosalva RE, Dyckman D, Melzer JM. Delayed facial nerve palsy after open reduction of an isolated zygomaticomaxillary complex fracture. J Craniofac Surg 2016;27(04):e392-e394

7 Xu P, Liu W, Zuo W, Wang D, Wang H. Delayed facial palsy after tympanomastoid surgery: A report of 15 cases. Am J Otolaryngol 2015;36(06):805-807

8 Magliulo G, D’Amico R, Di Cello P. Delayed facial palsy after vestibular schwannoma resection: clinical data and prognosis. J Otolaryngol 2003;32(06):400-404

9 Franco-Vidal V, Nguyen DQ, Guerin J, Darrouzet V. Delayed facial paralysis after vestibular schwannoma surgery: role of herpes viruses reactivation-our experience in eight cases. Otol Neurotol 2004;25(05):805-810

10 Scheller C, Strauss C, Fahlbusch R, Romstöck J. Delayed facial nerve paresis following acoustic neuroma resection and postoperative vasoactive treatment. Zentralbl Neurochir 2004;65(03):103-107

11 Carlstrom LP, Copeland WR III, Neff BA, Castner ML, Driscoll CL, Link $\mathrm{MJ}$. Incidence and risk factors of delayed facial palsy after vestibular schwannoma resection. Neurosurgery 2016;78(02):251-255

12 Morton RP, Ackerman PD, Pisansky MT, et al. Prognostic factors for the incidence and recovery of delayed facial nerve palsy after vestibular schwannoma resection. J Neurosurg 2011;114(02):375-380

13 House JW, Brackmann DE. Facial nerve grading system. Otolaryngol Head Neck Surg 1985;93(02):146-147

14 Sargent EW, Kartush JM, Graham MD. Meatal facial nerve decompression in acoustic neuroma resection. Am J Otol 1995;16(04): 457-464

15 Holliday MJ, Sampath P. Decompression of the labyrinthine segment of the facial nerve in acoustic neuroma surgery: a consideration for minimizing postoperative delayed facial nerve dysfunction. Neurosurg Focus 1998;5(03):e7

16 Scheller C, Richter HP, Engelhardt M, Köenig R, Antoniadis G. The influence of prophylactic vasoactive treatment on cochlear and facial nerve functions after vestibular schwannoma surgery: a prospective and open-label randomized pilot study. Neurosurgery 2007;61(01):92-97, discussion 97-98

17 Scheller C, Wienke A, Tatagiba M, et al. Interobserver variability of the House-Brackmann facial nerve grading system for the analysis of a randomized multi-center phase III trial. Acta Neurochir (Wien) 2017;159(04):733-738 